\title{
Small and nearly isotropic hole-like Fermi surfaces in LiFeAs detected through de Haas-van Alphen effect
}

\author{
B. Zeng \\ D. Watanabe \\ Q. R. Zhang \\ G. Li
}

Tiglet Besara

See next page for additional authors

Follow this and additional works at: https://bearworks.missouristate.edu/articles-cnas

\section{Recommended Citation}

Zeng, Bin, Daiki Watanabe, Q. R. Zhang, Gang Li, Tiglet Besara, Theo Siegrist, L. Y. Xing et al. "Small and nearly isotropic hole-like Fermi surfaces in LiFeAs detected through de Haas-van Alphen effect." Physical Review B 88, no. 14 (2013): 144518.

This article or document was made available through BearWorks, the institutional repository of Missouri State University. The work contained in it may be protected by copyright and require permission of the copyright holder for reuse or redistribution.

For more information, please contact BearWorks@library.missouristate.edu. 


\section{Authors}

B. Zeng; D. Watanabe; Q. R. Zhang; G. Li; Tiglet Besara; T. Siegrist; L. Y. Xing; X. C. Wang; C. Q. Jin; and For complete list of authors, see publisher's website. 


\title{
Small and nearly isotropic hole-like Fermi surfaces in LiFeAs detected through de Haas-van Alphen effect
}

\author{
B. Zeng, ${ }^{1}$ D. Watanabe,,${ }^{1,2}$ Q. R. Zhang, ${ }^{1}$ G. Li ${ }^{1}$ T. Besara, ${ }^{1}$ T. Siegrist,${ }^{1,3}$ L. Y. Xing, ${ }^{4}$ X. C. Wang, ${ }^{4}$ \\ C. Q. Jin, ${ }^{4}$ P. Goswami, ${ }^{1}$ M. D. Johannes, ${ }^{5}$ and L. Balicas ${ }^{1, *}$ \\ ${ }^{1}$ National High Magnetic Field Laboratory, Florida State University, Tallahassee, Florida 32310, USA \\ ${ }^{2}$ Department of Physics, Kyoto University, Kyoto 606-8502, Japan \\ ${ }^{3}$ Department of Chemical and Biomedical Engineering, Florida State University, Tallahassee, Florida 32310, USA \\ ${ }^{4}$ Institute of Physics, Chinese Academy of Sciences, Beijing 100190, China \\ ${ }^{5}$ Center for Computational Materials Science, Naval Research Laboratory, Washington, DC 20375, USA \\ (Received 29 September 2013; revised manuscript received 16 October 2013; published 30 October 2013)
}

\begin{abstract}
LiFeAs is unique among the arsenic based Fe-pnictide superconductors because it is the only nearly stoichiometric compound which does not exhibit magnetic order. This is at odds with electronic structure calculations which find a very stable magnetic state and predict cylindrical hole- and electron-like Fermi surface sheets whose geometry suggests spin fluctuations and a possible instability toward long-range ordering at the nesting vector. In fact, a complex magnetic phase diagram is indeed observed in the isostructural NaFeAs compound. Previous angle-resolved photoemission (ARPES) experiments revealed the existence of both hole and electron-like surfaces, but with rather distinct cross-sectional areas and an absence of the nesting that is thought to underpin both magnetic order and superconductivity in the pnictide family of superconductors. These ARPES observations were challenged by subsequent de Haas-van Alphen (dHvA) measurements which detected a few, electron-like Fermi surface sheets in rough agreement with the original band calculations. Here, we show a detailed dHvA study unveiling additional, small and nearly isotropic Fermi surface sheets in LiFeAs single crystals, which ought to correspond to hole-like orbits, as previously observed by ARPES. Therefore, our results reconcile the apparent discrepancy between ARPES and the previous dHvA results. The small size of these Fermi surface pockets suggests a prominent role for the electronic correlations in LiFeAs. The absence of gap nodes, in combination with the coexistence of quasi-two-dimensional and three-dimensional Fermi surfaces, favor an $s$-wave pairing symmetry for LiFeAs. But similar electron-like Fermi surfaces combined with very different hole pockets between $\mathrm{LiFeAs}$ and $\mathrm{LiFeP}$ suggest that the nodes in the gap function of LiFeP might be located on the hole pockets. This would be difficult to reconcile with the current understanding of the $s \pm$ scenario.
\end{abstract}

DOI: $10.1103 /$ PhysRevB.88.144518

PACS number(s): 74.70.Xa, 74.25.Dw, 74.62.Dh, 74.25.fc

\section{INTRODUCTION}

The uniqueness of LiFeAs among the Fe pnictides stems from the simple fact that it is a nearly stoichiometric superconductor: Previous studies indicate a molar ratio for $\mathrm{Li} / \mathrm{Fe} / \mathrm{As}$ of 0.99:1.00:1.00, through inductively coupled plasma mass spectrometry. ${ }^{1-3}$ In contrast to most Fe-based superconductors, in LiFeAs very small deviations in stoichiometry such as excess $\mathrm{Fe}$, suppress superconductivity very quickly: $\mathrm{LiFe}_{1.01} \mathrm{As}$ would no longer be superconducting according to the detailed structural analysis of Refs. 4 and 5.

Our band structure calculations, like earlier ones, ${ }^{6,7}$ indicate the existence of three hole-like Fermi surface sheets in LiFeAs, i.e., a small elongated ellipsoid and two corrugated cylinders around the $\Gamma$ point of the Brillouin zone, as well as two electron-like corrugated cylinders around its $M$ point, see Fig. 1. As with the parent compounds of other Fe-based superconductors, the calculated magnetic ground state is energetically favored over a nonmagnetic one, and the calculated cross-sectional areas of the large hole- and electron-like cylinders are very similar. In combination, these features point to a magnetic instability that must be suppressed (in general by doping or pressure) before the superconducting state can emerge. Instead, LiFeAs has a superconducting ground state with $T_{c} \sim 18 \mathrm{~K}$, a situation comparable to LiFeP which has a remarkably similar Fermiology and $T_{c}=5 \mathrm{~K}$.
Furthermore, although a number of experiments indicate a fully gapped as well as multigapped superconducting state for LiFeAs,${ }^{8-13}$ penetration depth measurements unveil nodal lines for the gap function of LiFeP. ${ }^{8}$ Earlier angle-resolved photoemission experiments (ARPES) ${ }_{1}^{14}$ revealed the existence of two-hole-like cylinders at the $\Gamma$ point, with quite distinct cross-sectional areas compared to those of the electron sheets at the $M$ point. This weak "nesting" between hole- and electron-like Fermi surface sheets would explain the absence of long range antiferromagnetism, but instead generate antiferromagnetic fluctuations, which are a plausible ingredient for the superconducting pairing mechanism. Subsequent ARPES studies ${ }^{13,15}$ confirmed these observations, and unveiled fully gapped superconducting gaps in all four FS sheets, with some of the gaps displaying a strong anisotropy in $k$ space. ${ }^{13}$

Therefore, it came as a surprise that a subsequent investigation on the de Haas-van Alphen effect, on both LiFeP and LiFeAs, found a good agreement with density functional theory (DFT) calculations. ${ }^{16}$ Although, in LiFeAs only three of the possible ten orbits were observed, and attributed to orbits on the electron-like sheets. To address this apparent discrepancy concerning the size of the hole-like Fermi surfaces, a couple of reports ${ }^{15,17}$ performed a dynamical mean field theory (DMFT) study, to introduce the effect of electronic correlations on the band structure and on the concomitant Fermi surfaces resulting from the DFT calculations. Several studies ${ }^{15,17-19}$ 
(a)

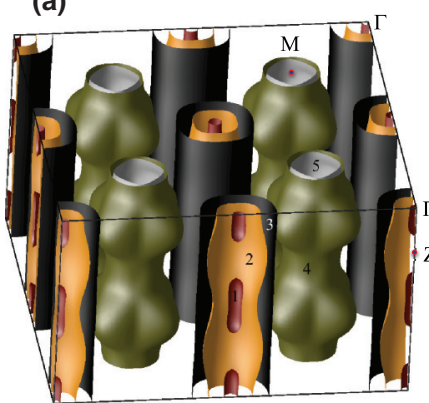

(b)

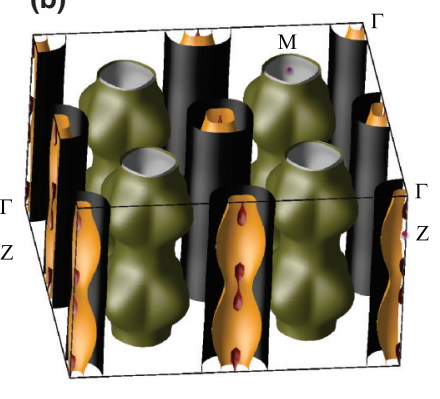

FIG. 1. (Color online) (a) Fermi surface of LiFeAs according to density functional theory calculations. (b) Same as in (a) after a shift of the Fermi level. Notice how the the hole-like Fermi surface sheets around the $\Gamma$ point shrink in size when one increases the electron count.

indicate that the correlations are mainly controlled by the value of the Hund's rule coupling $J$. Hund's coupling was found to shrink the middle hole pocket which has $t_{2 g}, d_{x z}$, and $d_{y z}$ orbital character, leaving the electron FS sheets intact. ${ }^{17}$ The fact that correlations tend to weaken, or even suppress, the nesting between FSs in LiFeAs, is confirmed by the DFT-DMFT study of Ref. 15. It finds that correlations affect the hybridization magnitude between the orbitals, resulting in a net transfer of charges from the $d_{x y}$ to the $d_{x z} / d_{y z}$ orbitals. This results in a downshift of the $d_{x z} / d_{y z}$ bands and in an upshift of $d_{x y}$-related bands with a concomitant decrease (increase) in the size of the hole FSs having $d_{x z} / d_{y z}\left(d_{x y}\right)$ character. Similarly, Ref. 15 finds practically no effect of the correlations on the geometry of the electron-like FSs.

Here we unveil a study on the de Haas-van Alphen effect (dHvA) on LiFeAs single crystals revealing a series of small extremal Fermi surface cross-sectional areas, in addition to the previously observed electron-like ones. ${ }^{16}$ These orbits can only be attributed to the two inner hole-like surfaces, not previously seen by dHvA. We find evidence for an orbit whose area matches the DFT prediction for the innermost hole pocket. However, and surprisingly, it is observed to be far less anisotropic than the DFT prediction, seen in Fig. 1. We also detect another set of smaller frequencies, i.e., 330 and $460 \mathrm{~T}$, which can only correspond to the predicted, middle hole-like Fermi surface around the $\Gamma$ point. An estimate of the cross-sectional area for this FS based on previous ARPES measurements ${ }^{14}$ yields a value of $3.215 \times 10^{-3} \AA^{-2}$ which is nearly perfectly matched by the Onsager cross-sectional area $(A=2 \pi e F / \hbar)$ related to the frequency $F=330 \mathrm{~T}$, or $3.15 \times 10^{-3} \AA^{-2}$. Therefore, and in agreement with the ARPES results, its size is considerably smaller than the DFT prediction implying that the electronic structure of LiFeAs is severely affected by electronic correlations (following the above DMFT arguments). Even more remarkable is the fact that these orbits are also nearly three dimensional. In general, the DFT approximation to the exchange correlation functional predicts larger metallic bandwidths than those observed experimentally, ${ }^{20}$ thus it yields comparatively less anisotropic Fermi surfaces. It is therefore intriguing that our experimental results show a strong renormalization of the calculated electronic masses (or bandwidths), together with
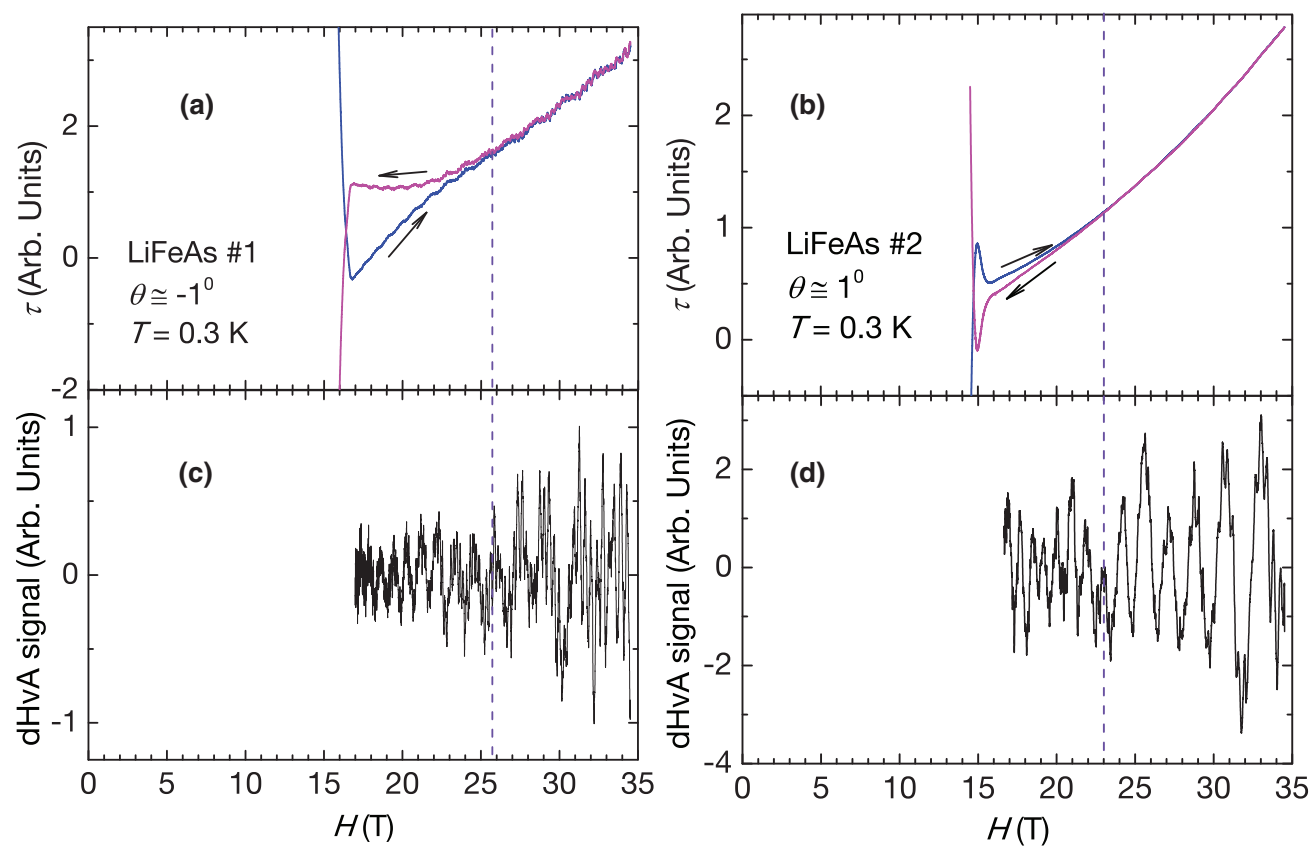

FIG. 2. (Color online) (a) Magnetic torque $\tau$ as a function of the magnetic field $H$ for a LiFeAs single crystal (crystal 1 ) at a temperature $T=0.3 \mathrm{~K}$ and an angle $\theta \simeq 1^{\circ}$ between $H$ and the interplanar $c$ axis. Blue and magenta lines indicate increasing and decreasing field sweeps. Notice how the large (and incomplete) hysteresis loop is followed at higher fields by a smaller one as previously seen in Ref. 23. (b) Same as in (a) but for a second LiFeAs single crystal (crystal 2). (c) The oscillatory component, or the de Haas-van Alphen effect, superimposed onto the $\tau(H)$ trace shown in (a) after the subtraction of a polynomial fit, and as a function of $H$. Vertical purple line indicates the irreversibility field. Hence, the quantum oscillatory phenomena is observed deep inside the irreversible superconducting region. (d) Same as in (c) but for the torque data in (b). Notice how the irreversibility field is higher for sample 1 when compared to 2, therefore indicating a higher sample quality. 
a simultaneous increase in Fermi surface isotropy in contrast to our calculations. We conclude that our results suggest that the nodes in the gap function of $\mathrm{LiFeP}^{8}$ might be located on the hole pockets, given that the electron-like FSs of LiFeAs and LiFeP are very similar in size and geometry, but the size of the hole pockets in LiFeAs are considerably different. Coupled to nearly isotropic Fermi surface sheets, this would also suggest a pairing symmetry possibly distinct from the originally proposed $s^{ \pm}$scenario for $\mathrm{Fe}$ pnictide superconductors. ${ }^{7}$

\section{EXPERIMENTAL RESULTS}

High-quality single crystals of LiFeAs have been grown by a self-flux technique. ${ }^{21,22}$ The precursor of $\mathrm{Li}_{3} \mathrm{As}$ was synthesized from Li pieces and As chips that were sealed in a $\mathrm{Nb}$ tube under $\mathrm{Ar}$ atmosphere and then treated at $650^{\circ} \mathrm{C}$ for $15 \mathrm{~h}$ in a sealed quartz tube. The $\mathrm{Li}_{3} \mathrm{As}, \mathrm{Fe}$, and As powders were mixed using the following ratio: $\mathrm{Li}: \mathrm{Fe}: \mathrm{As}=1: 0.3: 1$. The powder mixture was then pressed into a pellet in an alumina oxide tube. The sealed quartz tube was heated at $800{ }^{\circ} \mathrm{C}$ for $10 \mathrm{~h}$ before heating up to $1100^{\circ} \mathrm{C}$ at which it was held for another $10 \mathrm{~h}$. Finally, it was cooled down to $800^{\circ} \mathrm{C}$ at a rate of $2{ }^{\circ} \mathrm{C}$ per hour. Crystals with a size up to $4 \mathrm{~mm} \times 3 \mathrm{~mm}$ $\times 0.5 \mathrm{~mm}$ were obtained. The whole preparation work was performed within a glove box (or in high purity Ar gas). Torque magnetometry was measured by using the capacitive method with a $0.0762 \mathrm{~mm}$ thick $\mathrm{CuBe}$ lever. The angle between the sample and the external field was measured with a Hall probe.

Figure 2 shows the magnetic torque $\tau$ as a function of the magnetic field (as well as the oscillatory component superimposed onto it, or the dHvA effect) for two LiFeAs single crystals named here thereafter as crystals 1 and 2, respectively at $T=0.5 \mathrm{~K}$ and $T=0.3 \mathrm{~K}$ and for fields nearly aligned along the $c$ axis. The blue line represents increasing field scan, while the magenta line represents the decreasing field one. The large hysteresis corresponds to the superconducting signal, which is dominated by vortex pinning, and is several orders of magnitude larger $\left(>10^{3}\right)$ than the signal in provenance from the paramagnetic metallic state. Notice how the original (incomplete) hysteresis loop is followed by a much smaller one, where the lower branch becomes the upper one, and the upper one becomes the lower branch. Such a behavior, suggesting a crossover from a net diamagnetic response due to vortex pinning, towards an enhanced paramagnetic-like and hysteretic response (within the superconducting state), was already reported by us in Ref. 23 and attributed to a possible field-induced chiral superconducting state in LiFeAs. We emphasize that we studied well over a dozen crystals, and as clearly seen here, this anomalous hysteretic response is only observed on those crystals with enough purity to display the de Haas-van Alphen effect. The quality of the crystal can be judged, for example, through the value of the irreversibility field $H_{\text {irr }}$ (or the value in field where the hysteresis loop closes), which for crystal 1 is $\sim 26 \mathrm{~T}$, i.e., considerably higher than the values of $H_{c 2}$ previously reported for LiFeAs at this temperature and for this field orientation. ${ }^{24-26}$ Notice also that the dHvA effect is observed deep inside the irreversible superconducting region, i.e., from $H \lesssim 17 \mathrm{~T}$ up to $H_{\text {irr }} \sim 26 \mathrm{~T}$.

Figure 3 shows the oscillatory signal, or the dHvA effect, superimposed onto the torque traces acquired at $T=0.5 \mathrm{~K}$ for LiFeAs single-crystal 1 as a function of the inverse field $H^{-1}$ and for two values of the angle $\theta=-1^{\circ}$ and $7^{\circ}$, respectively. The dHvA signal is obtained from $\tau(H, T)$ after
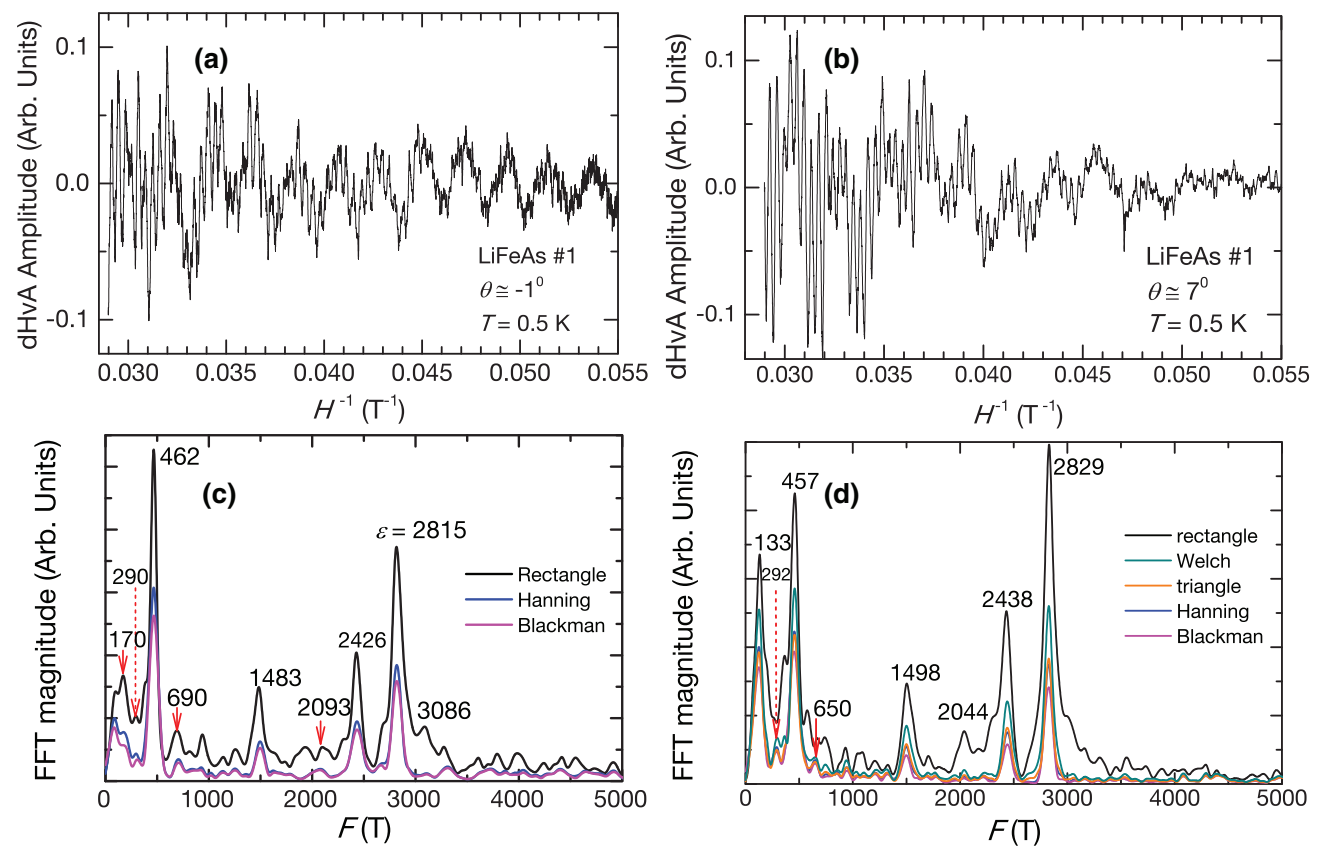

FIG. 3. (Color online) (a) Oscillatory component superimposed onto the torque signal obtained at $T=0.5 \mathrm{~K}$ from crystal 1 as a function of the inverse magnetic field $H^{-1}$ and for an angle $\theta \simeq-1^{\circ}$ between $H$ and the interlayer $c$ axis. (b) Same as in (a) but for an angle $\theta=7^{\circ}$. Notice how the position of the main peaks in frequency is independent of the selected spectral window. (c) Fast Fourier transform of the oscillatory signal shown in (a) for several spectral windows. (d) Same as in (c) but for $\theta=7^{\circ}$. 

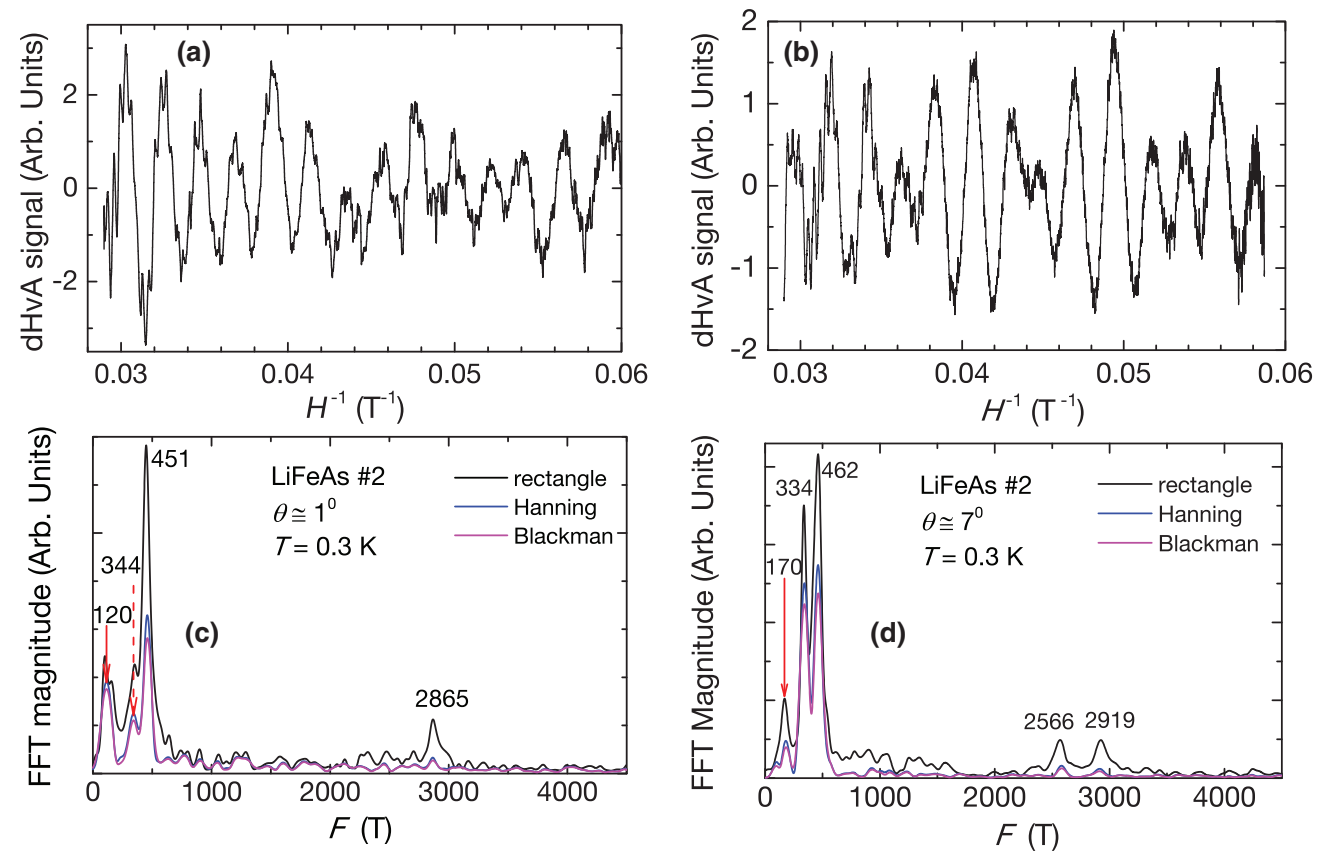

FIG. 4. (Color online) (a) Oscillatory component superimposed onto the torque signal obtained from crystal 2 at $T=0.3 \mathrm{~K}$ as a function of the inverse magnetic field $H^{-1}$ and for an angle $\theta \simeq 1^{\circ}$. (b) Same as in (a) but for an angle $\theta=7^{\circ}$. (c) Fast Fourier transform of the oscillatory signal shown in (a) for several spectral windows. Same as in (c) but for $\theta=7^{\circ}$.

subtraction of a polynomial background. Figure 3 also shows the fast Fourier transform (FFT) for each trace, and for several spectral windows. Notice how the amplitude of certain peaks observed when using a rectangular spectral window [2093 and 2044 T, or 690 and 650 T in Figs. 3(c) and 3(d), respectively] disappear towards the level of the background signal when different spectral windows are used. This indicates that they are not intrinsic frequencies but an artifact resulting from spectral leakage. Regardless of the chosen spectral window, two sharp peaks are observed at $F_{\delta} \simeq 2400 \mathrm{~T}$ and $F_{\epsilon} \simeq 2800 \mathrm{~T}$ which were previously reported in Ref. 16 and attributed to orbits on both electron-like Fermi surface sheets: neck and "belly" cyclotron orbits, respectively, on the gray and green FS sheets shown in Fig. 1(a). We also observed a pronounced, spectral window independent peak around $F \simeq 460 \mathrm{~T}$ which is preceded by a smaller peak at $F \simeq 290 \mathrm{~T}$. These values are relatively close to the frequencies for the $\alpha$ orbits predicted by the DFT calculations for the innermost hole-like FS sheet in LiFeP, but are clearly at odds with the DFT calculations for LiFeAs. Given their small size, one must assume that these frequencies correspond to orbits on hole FSs. We also detect evidence for another pair of smaller frequencies having very close values, respectively $\sim 130$ and $170 \mathrm{~T}$, which coincidentally are very close to the orbits/frequencies predicted by DFT for the innermost hole-like FS of LiFeAs, i.e., 121 and $132 \mathrm{~T}$. These values should be taken with a grain of salt, given that the amplitude and even the precise value of such small frequencies can be easily affected by the background subtraction. Finally, we observe another peak at $F=1500 \mathrm{~T}$ which one could be tempted to attribute to the electron-like $F_{\beta}=1590 \mathrm{~T}$ orbit reported in Ref. 16 .

Given the discrepancy between the DFT calculations and the cross-sectional areas observed by us in sample
1 , it is important to verify the reproducibility of these orbits/frequencies on a second single crystal. Therefore, Fig. 4 shows the oscillatory signal superimposed onto the torque measurements performed on a second crystal (LiFeAs 2) as a function of $H^{-1}$ for $T=0.3 \mathrm{~K}$, and for two angles, i.e., $\theta=1^{\circ}$ and $7^{\circ}$, respectively. The same Fig. 3 also shows the respective FFTs for several spectral windows, reproducing all the frequencies/orbits (within a margin of 10\%) previously observed in crystal 1 except for the frequency $F \simeq 1500 \mathrm{~T}$. Consequently, one can state with confidence that these small frequencies are intrinsic to LiFeAs, and given their small size, they ought to correspond to small hole pockets not seen in the previous dHvA study. ${ }^{16}$ As stated earlier, if one just takes the small inner hole orbit observed by ARPES, which is depicted

TABLE I. Fermi surface orbits, related dHvA frequencies, and band masses as predicted by the DFT calculations, compared to the experimentally observed dHvA frequencies, effective masses $m^{\star}$, and effective mass enhancements $\lambda=\frac{m^{\star}}{m_{b}}-1$.

\begin{tabular}{lrrrrrrr}
\hline \hline & \multicolumn{3}{c}{ DFT } & & \multicolumn{5}{c}{ Experiment } \\
\cline { 1 - 4 } \cline { 5 - 7 } Orbit & $F(T)$ & $m_{b}$ & & Orbit & $F(T)$ & $m^{\star}$ & $\frac{m^{\star}}{m_{b}}-1$ \\
\hline $1_{a}$ & 121 & -0.33 & $\alpha_{a}$ & 120 & $(1.8 \pm 0.5)$ & 4.45 \\
$1_{b}$ & 132 & -0.27 & $\alpha_{b}$ & 170 & $(1.5 \pm 0.3)$ & 3.7 \\
$2_{a}$ & 1561 & -2.24 & $\zeta_{a}$ & 330 & $(4.1 \pm 0.5)$ & 0.83 \\
$2_{b}$ & 2477 & -1.55 & $\zeta_{b}$ & 460 & $(0.8 \pm 0.1)$ & -0.48 \\
$3_{a}$ & 4597 & -2.18 & & & & \\
$4_{a}$ & 2392 & 1.19 & $\delta$ & 2400 & $(4.4 \pm 0.7)$ & 2.7 \\
$4_{b}$ & 6300 & 2.39 & & & & \\
$5_{a}$ & 1590 & 1.58 & & $\beta^{\sharp}$ & & & \\
$5_{b}$ & 2974 & 0.99 & $\epsilon$ & 2800 & $(4.4 \pm 0.5)$ & 3.44 \\
\hline \hline
\end{tabular}


(a)

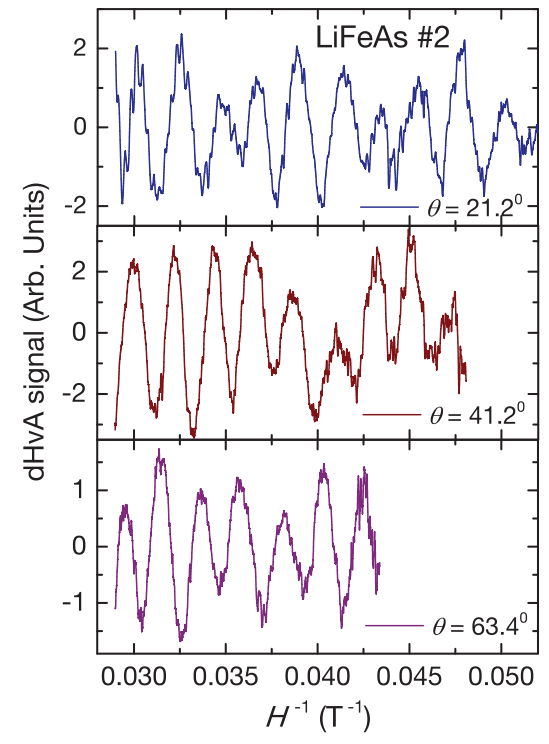

(b)

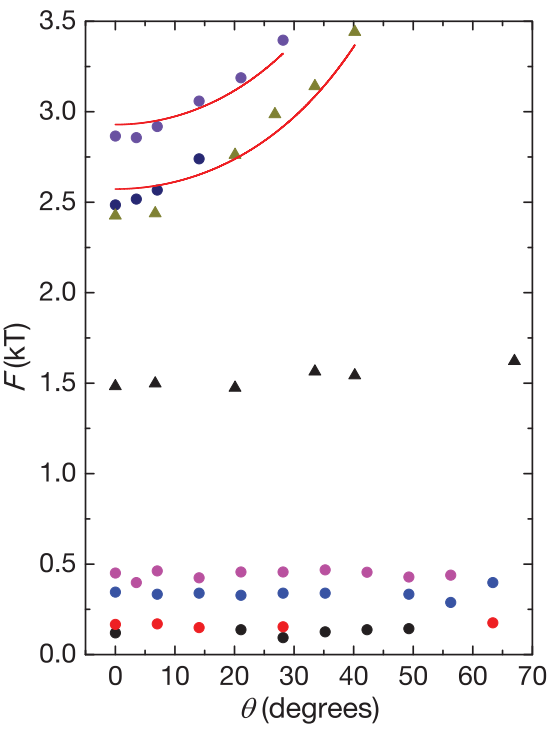

(c)

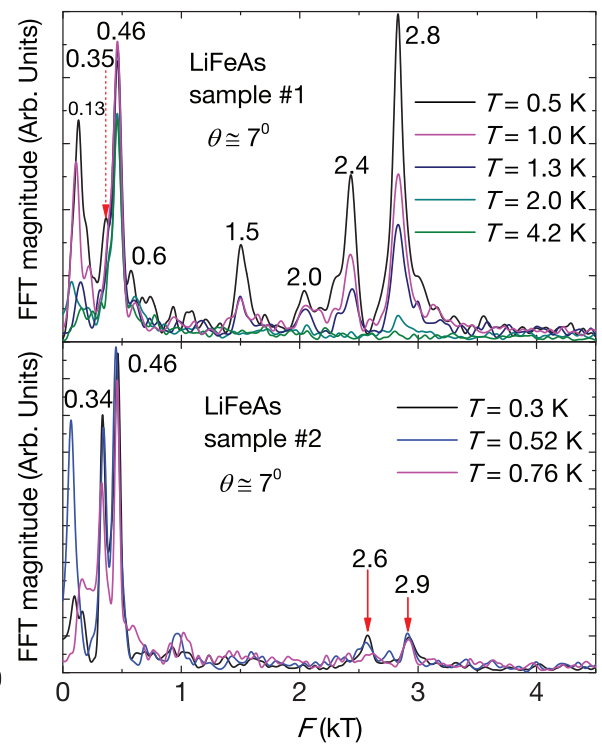

FIG. 5. (Color online) (a) Oscillatory component superimposed onto the torque signal obtained from sample 2 , at $T=0.3 \mathrm{~K}$, and for several angles $\theta$. (b) Angular dependence of several of the frequencies observed in the FFT spectra. Red lines are fits to $F=F_{0} / \cos (\theta)$, i.e., the angular dependence expected for two-dimensional cross-sectional areas. Notice how the smaller frequencies are nearly independent on angle. Therefore, open two-dimensional Fermi surface sheets coexist with closed and nearly isotropic sheets in LiFeAs. Notice that the orbit associated with the frequency $F \simeq 1500$ T observed only in sample 1 (black markers) would also be nearly isotropic. (c) Magnitude of the fast Fourier transform of the $\mathrm{dHvA}$ signal as a function of the frequency $F$, for several temperatures and for samples 1 and 2 , respectively. Here a rectangular spectral window was used.

in Fig. 1 of Ref. 27, one obtains a rough estimate for its area of just $\sim 0.12 \%$ of the area of the first-Brillouin zone in the $k_{x} k_{y}$ plane. It is equivalent to a frequency $F \simeq 338 \mathrm{~T}$ when using the Onsager relation. This frequency is remarkably close to the position of one of the main peaks seen in Figs. 4(c) and $4(\mathrm{~d})$ at $F$ of $\sim 330$ to $\sim 345 \mathrm{~T}$, respectively. The agreement between ARPES and our $\mathrm{dHvA}$ results is consistent with DFT calculations predicting a neutral surface for LiFeAs after cleaving, and the absence of a distinct electronic structure at its surface. ${ }^{28}$ A summary of our dHvA results as well as a comparison with the DFT calculations is presented in Table I. We have chosen not to include the orbit yielding $F=$ $1500 \mathrm{~T}$ observed from crystal 1 because it was not reproduced by the measurements on crystal 2 . Notice the remarkable quasiparticle mass enhancement for the innermost hole orbits.

Figure 5(a) shows the oscillatory signal superimposed on the torque response of crystal 2 at $T=0.3 \mathrm{~K}$ as a function of $H^{-1}$ and for several angles $\theta$. As seen, at larger angles the dHvA signal is dominated by the small frequencies. The irreversibility field increases quickly as the field is oriented towards the $a b$ plane, limiting our ability to access the metallic state and therefore the dHvA signal for angles beyond $\theta \gtrsim 70^{\circ}$. In Fig. 5(b) we display the angular dependence of the dHvA frequencies as extracted from both samples. As expected, the frequencies $F_{\delta}=2400 \mathrm{~T}$ and $F_{\epsilon}=2800 \mathrm{~T}$, attributed to electron-like Fermi surfaces, follow the $F=F_{0} / \cos (\theta)$ dependence (red lines) expected for two-dimensional orbits. Remarkably, the smaller orbits such as $\alpha_{a}$ or $\zeta_{a}$ do not display much angular dependence at all, implying that these are closed, nearly isotropic orbits. The $\alpha$ orbits were predicted to be three dimensional but very anisotropic, see Fig. 1 and
Refs. 6 and 16. On the contrary, the $\zeta$ orbits were predicted to be two dimensional. Perhaps this discrepancy between experiment and calculations might be addressed by shifting the Fermi level in the DFT calculations, which as seen in Fig. 1(b) would lead to smaller hole-like Fermi surfaces, and combining it with electronic correlations as in the DMFT approach of Refs. 15 and 17. Finally, Fig. 5(c) shows the magnitude of the FFT spectra for both crystals and for several temperatures at an angle $\theta \simeq 7^{\circ}$. From these traces we obtained the magnitude of each peak in the FFT spectra as a function of the temperature and extracted the effective masses (which are shown in Table I) through the Lifshitz-Kosevich formula: $x / \sinh x$ with $x=14.69 m^{\star} T / H$ and $m^{\star}$ as the effective mass in units of the free electron mass.

\section{DISCUSSION}

LiFeAs was claimed to be placed in the electron overdoped regime $^{3}$ due to series of observations: (i) An extreme sensitivity of the superconducting state with respect to Li deficiencies which play a role akin to $\mathrm{Zn}$ impurities ${ }^{29}$ in the overdoped regime of $\mathrm{LaFeAsO}_{1-x} \mathrm{~F}_{x}$, (ii) low-energy, transverse incommensurate spin excitations by neutron scattering experiments, ${ }^{3}$ and (iii) a very asymmetric line in the tunneling spectra, ${ }^{30,31}$ similar to what is seen in electron overdoped $\mathrm{NaFe}_{1-x} \mathrm{Co}_{x} \mathrm{As}$ and in sharp contrast to optimally doped $\mathrm{NaFe}_{1-x} \mathrm{Co}_{x} \mathrm{As}^{32}$ If this were indeed the case, according to the $s \pm$ scenario, 7,33 one could expect LiFeAs to either display nodes, or at least a strong angular modulation in the superconducting gap function on the electron FSs. In effect, for electron overdoped 
$\mathrm{Ba}\left(\mathrm{Fe}_{1-x} \mathrm{Co}_{x}\right)_{2} \mathrm{As}_{2}$ both thermal conductivity ${ }^{34}$ and heat capacity measurements ${ }^{35}$ indeed reveal evidence for nodes in its gap function. Nevertheless, all the experimental evidence collected so far from LiFeAs points towards a fully gapped superconducting state, albeit with strong gap anisotropies. ${ }^{8,13}$ Remarkably, evidence for a nodal superconducting state was instead found in LiFeP which according to the experimental results shown here, and in agreement with other groups, $8,13,14,16$ has electron-like Fermi surfaces of nearly the same size as those of LiFeAs. Furthermore, according to our work and in agreement with ARPES, the main effect of replacing As with $\mathrm{P}$ is to severely shrink the middle hole FS and to expand the outer one. If one assumed the same pairing symmetry for both compounds, a natural assumption given their similar structure and chemical composition, the nodes in the superconducting gap function would have to be located on the hole-like Fermi surfaces of $\mathrm{LiFeP}$. It remains to be clarified whether this fact, coupled with the existence of two sets of nearly isotropic, three-dimensional hole-like Fermi surfaces in LiFeAs, can be reconciled with the $s \pm$ pairing symmetry claimed to address nearly all aspects of the superconducting state in the Fe pnitide/chalcogenide superconductors. ${ }^{33,36}$ Nonetheless, in LiFeAs the absence of nodes and the coexistence of three-dimensional with two-dimensional Fermi surface sheets, indicates a superconducting pairing symmetry with a sizable $s$ component. Finally, our results provide support to a recent magneto-transport study in LiFeAs which requires (within a three-band model) the existance of a small hole pocket although with extremely high-hole mobility. ${ }^{37}$

\section{ACKNOWLEDGMENTS}

We acknowledge useful discussions with A. Chubukov, Z. P. Yin, K. Haule, and P. J. Hirschfeld. L.B. is supported by DOE-BES through award DE-SC0002613. T.B. and T.S. are supported by DOE-BES through award DE-SC0008832, and by FSU. Funding for M.D.J. was provided by the Office of Naval Research (ONR) through the Naval Research Laboratory's Basic Research Program. The NHMFL is supported by NSF through NSF-DMR-0084173 and the State of Florida. *balicas@magnet.fsu.edu

${ }^{1}$ C. W. Chu, F. Chen, M. Gooch, A. M. Guloy, B. Lorenz, B. Lv, K. Sasmal, Z. J. Tang, J. H. Tapp, and Y. Y. Xue, Physica C 469, 326 (2009).

${ }^{2}$ I. Morozov, A. Boltalin, O. Volkova, A. Vasiliev, O. Kataeva, U. Stockert, M. Abdel-Hafiez, D. Bombor, A. Bachmann, L. Harnagea, M. Fuchs, H.-J. Grafe, G. Behr, R. Klingeler, S. Borisenko, C. Hess, S. Wurmehl, and B. Büchner, Cryst. Growth Design 10, 4428 (2010).

${ }^{3}$ M. Wang, M. Wang, H. Miao, S. V. Carr, D. L. Abernathy, M. B. Stone, X. C. Wang, L. Xing, C. Q. Jin, X. Zhang, J. Hu, T. Xiang, H. Ding, and P. Dai, Phys. Rev. B 86, 144511 (2012).

${ }^{4}$ M. J. Pitcher, T. Lancaster, J. D. Wright, I. Franke, A. J. Steele, P. J. Baker, F. L. Pratt, W. T. Thomas, D. R. Parker, S. J. Blundell, and S. J. Clarke, J. Am. Chem. Soc. 132, 10467 (2010).

${ }^{5}$ J. D. Wright, M. J. Pitcher, W. Trevelyan-Thomas, T. Lancaster, P. J. Baker, F. L. Pratt, S. J. Clarke, and S. J. Blundell, Phys. Rev. B 88, 060401(R) (2013).

${ }^{6}$ D. J. Singh, Phys. Rev. B 78, 094511 (2008).

${ }^{7}$ I. I. Mazin, D. J. Singh, M. D. Johannes, and M. H. Du, Phys. Rev. Lett. 101, 057003 (2008).

${ }^{8}$ K. Hashimoto, S. Kasahara, R. Katsumata, Y. Mizukami, M. Yamashita, H. Ikeda, T. Terashima, A. Carrington, Y. Matsuda, and T. Shibauchi, Phys. Rev. Lett. 108, 047003 (2012).

${ }^{9}$ F. Wei, F. Chen, K. Sasmal, B. Lv, Z. J. Tang, Y. Y. Xue, A. M. Guloy, and C. W. Chu, Phys. Rev. B 81, 134527 (2010).

${ }^{10}$ U. Stockert, M. Abdel-Hafiez, D. V. Evtushinsky, V. B. Zabolotnyy, A. U. B. Wolter, S. Wurmehl, I. Morozov, R. Klingeler, S. V. Borisenko, and B. Buchner, Phys. Rev. B 83, 224512 (2011).

${ }^{11}$ H. Kim, M. A. Tanatar, Y. J. Song, Y. S. Kwon, and R. Prozorov, Phys. Rev. B 83, 100502 (2011).

${ }^{12}$ M. A. Tanatar, J.-Ph. Reid, S. René de Cotret, N. Doiron-Leyraud, F. Laliberté, E. Hassinger, J. Chang, H. Kim, K. Cho, Y. J. Song, Y. S. Kwon, R. Prozorov, and L. Taillefer, Phys. Rev. B 84, 054507 (2011).
${ }^{13}$ K. Umezawa, Y. Li, H. Miao, K. Nakayama, Z.-H. Liu, P. Richard, T. Sato, J. B. He, D.-M. Wang, G. F. Chen, H. Ding, T. Takahashi, and S.-C. Wang, Phys. Rev. Lett. 108, 037002 (2012).

${ }^{14}$ S. V. Borisenko, V. B. Zabolotnyy, D. V. Evtushinsky, T. K. Kim, I. V. Morozov, A. N. Yaresko, A. A. Kordyuk, G. Behr, A. Vasiliev, R. Follath, and B. Büchner, Phys. Rev. Lett. 105, 067002 (2010).

${ }^{15}$ G. Lee, H. S. Ji, Y. Kim, C. Kim, K. Haule, G. Kotliar, B. Lee, S. Khim, K. H. Kim, K. S. Kim, K.S. Kim, and J. H. Shim, Phys. Rev. Lett. 109, 177001 (2012).

${ }^{16}$ C. Putzke, A. I. Coldea, I. Guillamón, D. Vignolles, A. McCollam, D. LeBoeuf, M. D. Watson, I. I. Mazin, S. Kasahara, T. Terashima, T. Shibauchi, Y. Matsuda, and A. Carrington, Phys. Rev. Lett. 108, 047002 (2012).

${ }^{17}$ J. Ferber, K. Foyevtsova, R. Valentí, and H. O. Jeschke, Phys. Rev. B 85, 094505 (2012).

${ }^{18}$ K. Haule and G. Kotliar, New J. Phys. 11, 025021 (2009).

${ }^{19}$ Z. P. Yin, K. Haule, and G. Kotliar, Nat. Mater. 10, 932 (2011).

${ }^{20}$ J. Paier, M. Marsman, K. Hummer, G. Kresse, I. C. Gerber, and J. G. Angyan, J. Chem. Phys. 124, 154709 (2006).

${ }^{21}$ X. C. Wang, Q. Q. Liu, Y. X. Lv, Z. Deng, K. Zhao, R. C. Yu, J. L. Zhu, and C. Q. Jin, Sci. China Phys. Mech. 53, 1199 (2010).

${ }^{22}$ X. C. Wang, Q. Q. Liu, Y. X. Lv, W. B. Gao, L. X. Yang, R. C. Yu, F. Y. Li, and C. Q. Jin, Solid State Commun. 148, 538 (2008).

${ }^{23}$ G. Li, R. R. Urbano, P. Goswami, C. Tarantini, B. Lv, P. Kuhns, A. P. Reyes, C. W. Chu, and L. Balicas, Phys. Rev. B 87, 024512 (2013).

${ }^{24}$ S. Khim, B. Lee, J. W. Kim, E. S. Choi, G. R. Stewart, and K. H. Kim, Phys. Rev. B 84, 104502 (2011).

${ }^{25}$ K. Cho, H. Kim, M. A. Tanatar, Y. J. Song, Y. S. Kwon, W. A. Coniglio, C. C. Agosta, A. Gurevich, and R. Prozorov, Phys. Rev. B 83, 060502(R) (2011).

${ }^{26}$ N. Kurita, K. Kitagawa, K. Matsubayashi, A. Kismarahardja, E.-S. Choi, J. S. Brooks, Y. Uwatoko, S. Uji, and T. Terashima, J. Phys. Soc. Jpn. 80, 013706 (2011).

${ }^{27}$ J. Knolle, V. B. Zabolotnyy, I. Eremin, S. V. Borisenko, N. Qureshi, M. Braden, D. V. Evtushinsky, T. K. Kim, A. A. Kordyuk, S. Sykora, 
Ch. Hess, I. V. Morozov, S. Wurmehl, R. Moessner, and B. Büchner, Phys. Rev. B 86, 174519 (2012).

${ }^{28}$ A. Lankau, K. Koepernik, S. Borisenko, V. Zabolotnyy, B. Büchner, J. van den Brink, and H. Eschrig, Phys. Rev. B 82, 184518 (2010).

${ }^{29}$ Y. Li, J. Tong, Q. Tao, C. Feng, G. Cao, W. Chen, F.-c. Zhang, and Z.-a. Xu, New J. Phys. 12, 083008 (2010).

${ }^{30}$ T. Hanke, S. Sykora, R. Schlegel, D. Baumann, L. Harnagea, S. Wurmehl, M. Daghofer, B. Büchner, J. van den Brink, and C. Hess, Phys. Rev. Lett. 108, 127001 (2012).

${ }^{31}$ S. Chi, S. Grothe, R. Liang, P. Dosanjh, W. N. Hardy, S. A. Burke, D. A. Bonn, and Y. Pennec, Phys. Rev. Lett. 109, 087002 (2012).
${ }^{32}$ X. Zhou, P. Cai, A. Wang, W. Ruan, C. Ye, X. Chen, Y. You, Z.-Y. Weng, and Y. Wang, Phys. Rev. Lett. 109, 037002 (2012).

${ }^{33}$ A. Chubukov, Annu. Rev. Condens. Matter Phys. 3, 57 (2012).

${ }^{34}$ J.-P. Reid, M. A. Tanatar, X. G. Luo, H. Shakeripour, N. DoironLeyraud, N. Ni, S. L. Bud'ko, P. C. Canfield, R. Prozorov, and L. Taillefer, Phys. Rev. B 82, 064501 (2010).

${ }^{35}$ D. J. Jang, A. B. Vorontsov, I. Vekhter, K. Gofryk, Z. Yang, S. Ju, J. B. Hong, J. H. Han, Y. S. Kwon, F. Ronning, J. D. Thompson, and T. Park, New J. Phys. 13, 023036 (2011).

${ }^{36}$ S. Maiti, M. M. Korshunov, and A. V. Chubukov, Phys. Rev. B 85, 014511 (2012).

${ }^{37}$ F. Rullier-Albenque, D. Colson, A. Forget, and H. Alloul, Phys. Rev. Lett. 109, 187005 (2012). 